\title{
Heterotopic ossification induced by hypoxia in a retrosternal gastric tube following transhiatal oesophagectomy
}

\author{
H C J L Buscher, J J B van Lanschot, A H Mulder, H W Tilanus
}

\begin{abstract}
A 71 year old man underwent retrosternal gastric tube reconstruction following transhiatal oesophagectomy for squamous cell carcinoma. On the second postoperative day, the patient developed a cardiac arythmia with secondary hypotension followed by hypoxaemia necessitating artificial ventilation. Two weeks after surgery, endoscopy revealed massive necrosis of the proximal segment of the gastric tube extending from the anastomosis in the neck to the watershed area. Three weeks later, the patient died and a necropsy was performed. Macroscopic evaluation of the gastric tube revealed a sharply demarcated and fully ossificated proximal segment. Heterotopic ossification was present on histological examination. This condition has only been described in conjunction with primary or metastatic gastric adenocarcinoma. The location of the ossification and the presence of temporary systemic hypoxia suggest that the latter was the main factor responsible for the ossificative response.

( $\mathcal{F}$ Clin Pathol 1995;48:177-178)
\end{abstract}

Keywords: Heterotopic ossification, oesophagus, anastomotic strictures.

Heterotopic ossification has to be clearly distinguished from calcification without osteoblastic activity. This condition has been described in many different tissues and is associated with several conditions. ${ }^{1}$ However, the underlying cause of heterotopic ossification is unclear. In the gastrointestinal tract the presence of heterotopic ossification is generally associated with cancerous or precancerous tissue. $^{23}$ Here, we describe ossification in a gastric tube in the absence of malignant tissue. Circumstantial evidence suggests that the heterotopic ossification was caused by severe local hypoxia.

\section{Case report}

A 71 year old man underwent transhiatal subtotal resection of the oesophagus for squamous cell carcinoma and retrosternal gastric tube reconstruction from an otherwise unimpaired normal stomach. At diagnosis, endoscopy did not reveal any abnormalities in the upper gastrointestinal tract besides the tumour in the oesophagus. On the second day following extubation, the patient developed a hemiplegia on the right side. Changes corresponding to transient lower wall ischaemia of the heart were detected. There were no signs of intracerebral bleeding on computed tomography. Nine days after surgery, the patient became unstable for a second time and had to be treated with inotropic agents. Endoscopy of the gastric tube was performed a day later and revealed an anastomotic dehiscence and ischaemic necrosis over $5 \mathrm{~cm}$ of the proximal gastric tube. Segmental necrosis was present and spread from the oesophagogastrostomy in the neck to a level which corresponds to the so-called watershed. The patient developed septicaemia complicated by respiratory insufficiency and deteriorated further. Endoscopy was repeated the day before the patient died. The necrotic segment was enlarged up to $10 \mathrm{~cm}$ distal to the anastomosis. Beyond this segment, the endoscope was unable to pass because of the presence of a stenosis at the border between the necrotic and normal mucosa. The patient died 23 days after surgery. Necropsy was performed after obtaining consent from the patient's family.

\section{Pathology}

On examination of the surgical specimen resected 23 days before the patient died, a moderately differentiated squamous cell carcinoma with a diameter of $3 \mathrm{~cm}$ was observed in the lower third of the oesophagus. The tumour had infiltrated the muscular wall and the perioesophageal connective tissue. The resection margins and the perioesophageal tissue were free of tumour, as were the lymph nodes $\left(\mathrm{T}_{3} \mathrm{~N}_{0} \mathrm{M}_{\mathrm{x}}\right)$. The oesophageal-gastric anastomosis appeared dehiscent. The proximal segment of the gastric tube was constricted over about $14 \mathrm{~cm}$ (fig 1). Erosions and ulcerations were present on the mucosal surface. The gastric wall had thickened and contained a gritty substance. On microscopic examination, the surface comprised necrotic tissue with a fibroblastic proliferation below. The muscularis propria was absent in some sections. A discontinuous layer of trabecular bone was present along the entire length of the constricted segment in the outer part of the gastric tube, extending into the adjacent mediastinal tissue. Ossification was most prominent in the proximal part and extended around the right carotid artery and close to the thyroid gland (fig 2). 


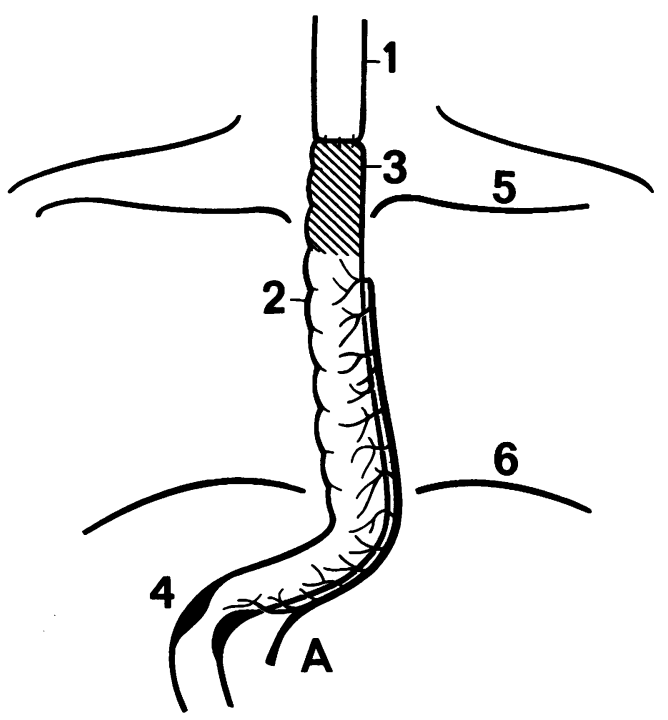

Figure 1 Schematic drawing of the gastric tube reconstruction. $1=$ cervical oesophagus; $2=$ gastric tube; $3=$ oesophagogastrostomy; $4=$ pylorus; $5=$ clavicula; $6=$ diaphragm; and $A=$ the right gastric epiploic artery. The shaded area corresponds with the ossificated segment.

The right gastric epiploic artery was present alongside the lower part of the gastric tube, but was absent from $1 \mathrm{~cm}$ below the constricted segment. Residual tumour was not present on necropsy. The gastric mucosa distal to the stenosis and ossification showed mild chronic gastritis.

\section{Discussion}

Dystrophic calcification and heterotopic ossification frequently occur following tissue injury. Heterotopic ossification has to be clearly distinguished from dystrophic calcification, which lacks osteoblastic activity.

Heterotopic ossification has been described in many different organs and in both neoplastic and non-neoplastic tissue. ${ }^{1}$ In non-neoplastic

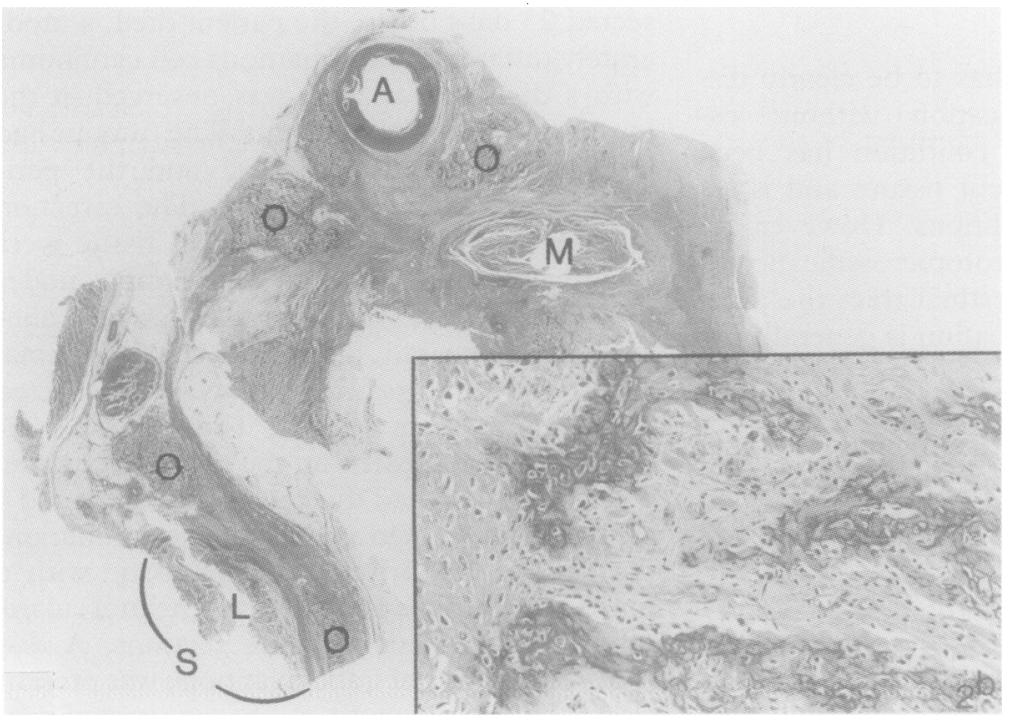

Figure 2 Transverse section of the upper mediastinum (decalcified and stained with haematoxylin and azophloxin). A=common cartid artery; $M=$ sternothyreoid muscle; $S=$ collapsed retrosternal gastric tube $(L=$ lumen $) ; O=$ ossified tissue. Insert: magnification of ossified tissue $(\times 118)$. tissue it is thought to be the mesenchymal cell response to altered intracellular metabolism or $\mathrm{pH}$ changes caused by physical, chemical, or infectious injury, ${ }^{45}$ or functional immobility in neurological trauma. ${ }^{1}$ Heterotopic ossification has also been reported in neoplastic tissue of epithelial origin and more specifically in the gastrointestinal tract. Osseous metaplasia of mesenchymal cells can be induced in specific circumstances. $^{2}$

Previously reported heterotopic ossification in the stomach was, in most cases, confined to primary tumours or metastatic lesions, under both clinical and experimental conditions. ${ }^{23}$ Only one experimental study reported heterotopic ossification following subtotal gastrectomy in rats. ${ }^{6}$ Bone formation, however, was not localised to the anastomosis but scattered in small foci in the mucosa of the glandular stomach.

In this case the heterotopic ossification was entirely restricted to the proximal part of a gastric tube adjacent to a well demarcated necrotic segment noted at gastroscopy. This area corresponds to the watershed area which normally receives blood from the left and right gastric epiploic arteries (fig 1). Only the right gastric epiploic artery remains following reconstruction of the gastric tube. ${ }^{7}$ Vascularisation of the proximal segment occurs through the submucosal microvascular network. ${ }^{8}$ Poor vascularisation of the gastric tube is an important risk factor for stricture formation, which occurs in 12 to $40 \%$ of patients following gastric tube reconstruction. ${ }^{9}$ Reportedly, massive gangrene of the gastric tube occurs in $1 \%$ of reconstructions. ${ }^{10}$ To our knowledge, however, heterotopic ossification has not been described previously in these circumstances.

Although the exact cause of the ossification in our patient remains unclear, its location and the presence of systemic hypoxia suggest that the latter was the main trigger.

1 An HS, Ebrahem N, Kim K, Jackson WT, Kane JT. Heterotopic ossification and pseudoarthrosis in the shoulder following encephalitis. A case report and review of the literature. Clin Orthop 1987;219:291-8.

2 Rhone DP, Horowitz RN. Heterotopic ossification in the pulmonary metastases of gastric adenocarcinoma. Report of a case and review of the literature. Cancer 1976.38. $1773-80$.

3 Werner B, Dahm K, Breucker H. Ossification in cancer of the stomach. An experimental study. Z Krebsforsch 1976; 86:147-54.

4 Ackerman LV. Extra osseous localized non-neoplastic bone and cartilage formation (so-called myositis ossificans). Clinical and pathological confusion with malignant neoplasms. F Bone foint Surg Am 1958;40A:279-98.

5 Yannopoulos K, Katz S, Flesher L, Geller A, Berrayo R. Mesenteritis ossificans. Am $\mathcal{f}$ Gastroenterol 1992;87:230-3.

6 Ackerman NB. Bone formation in the gastric mucosa following subtotal gastrectomy in rats. Exp Mol Pathol 1968; 9:125-30.

7 Akiyama H, Tsurumaru M, Kawamura T, Ono Y. Principles of surgical treatment for carcinoma of the esophagus. Analysis of lymph node involvement. Ann Surg 1981;194: 438-46.

8 Salo JA, Perhoniemi VJ, Heikkinen LO, Verkkala KA, Jarvinen AAJ. Pulse oximetry for the assessment of gastric vinen AAJ. Pulse oximetry for the assessment of gastric tube circulation

9 Pierie JPEN, Graaf de PW, Poen H, Tweel van der I, Obertop $H$. Incidence and management of benign anastomotic strictures following cervical oesophagogastrostomy. $\mathrm{Br} \mathscr{F}$ Surg 1993;80:471-4.

10 Moorehead RJ, Wong J. Gangrene in esophageal substitutes after resection and bypass procedures for carcinoma of the esophagus. Hepatogastroenterology 1990;37:364-7. 